\title{
Density Functional Partitions
}

\author{
John A. Pople* \\ Department of Chemistry, Northwestern University, Evanston, Illinois 60208
}

\author{
Ross D. Adamson and Peter M. W. Gill \\ Department of Chemistry, Massey University, Palmerston North, New Zealand \\ Received: February 15, $1996^{\otimes}$
}

The performance of the local spin density approximation (LSD) of Kohn-Sham density functional theory when applied to partitioned electron densities has been explored. Spin partitioning and core/valence partitioning have each been examined and the results compared with conventional Møller-Plesset (MP2) theory based on the Kohn-Sham determinant. It is found that the LSD energy contributions from such subsets of electrons are generally in poor agreement with their conventional counterparts, and it is proposed that such differences be employed as a diagnostic measure of the quality of other density functionals.

\section{Introduction}

A major target in density functional theory (DFT) is the development of a general electronic energy functional ${ }^{1}$ for exchange and correlation $E_{\mathrm{XC}}[\gamma]$, given a full one-electron density $\gamma$. Although no systematic procedure for improving such a functional is presently apparent, some progress is possible by comparing DFT techniques with more conventional methods of calculating exchange and correlation energies, using configuration interaction (or related perturbation or coupled-cluster theories), based on a single-determinant reference wave function. Such research has been advanced in recent years by the use of common features (orbital basis sets, integral evaluation algorithms, etc.) for combined conventional-DFT computer programs. $^{2}$ This has allowed some comparisons between DFT and conventional (Møller-Plesset, MP) correlation energies for some molecules. ${ }^{3}$

It would be helpful if DFT and conventional methods were compared in greater detail by examining their performance for various subsets of electrons in any molecule. Such partitions of the total correlation energy are common in conventional theory. For example, the correlation energy associated with inner-shell electrons is often separated and ignored (the so-called frozen-core approximation). Also, it is possible (particularly at the simplest MP2 level) to separate correlation between electrons of parallel $(\alpha \alpha+\beta \beta)$ and antiparallel $(\alpha \beta)$ spin. At the MP2 level, the total correlation energy is expressible as a simple sum of electron-pair components, so such partitions are straightforward.

The primary purpose of this paper is to attempt similar partitions of DFT exchange-correlation energies and enquire whether they correspond properly to their conventional counterparts. This is carried out by examining electron correlation relative to the Kohn-Sham (KS) single-determinant reference wave function. Such correlation energies may be obtained either from conventional theory or from DFT. Each may be partitioned by dividing the occupied spin orbitals into two nonoverlapping sets. This corresponds to a division of the density into two parts, so that the resulting energy components may be compared.

Our approach follows the earlier work of Stoll et al. ${ }^{4}$ and Perdew et al., ${ }^{5}$ and we confirm some of the conclusions of these

\footnotetext{
${ }^{\otimes}$ Abstract published in Advance ACS Abstracts, March 15, 1996.
}

workers. Because the results presented here are preliminary, we restrict our attention to the local spin density (LSD) level. We report detailed studies using more sophisticated levels elsewhere. ${ }^{6}$ After presenting the general theory in sections 2 and 3, we describe some preliminary applications to the LSD energy functional in sections 4 and 5. The results, already welldocumented for total correlation energies, are examined piecewise in both spin and core/valence partitions.

\section{General Theory}

The starting point of density functional theory is the electron density $\rho(\mathbf{r})$ where $\mathbf{r}$ are electronic Cartesian coordinates. In the spin-dependent form of the theory we shall use, there will be two components: $\rho^{\alpha}$ and $\rho^{\beta}$ for $\alpha$ and $\beta$ electrons, respectively. We shall find it convenient to use the symbol $\gamma(\mathbf{x})$ for the density in the full four-dimensional space, $\mathbf{x}$ representing Cartesian and spin coordinates for a single electron. This notation was introduced by Löwdin ${ }^{7}$ and followed, in part, in the book by Parr and Yang. ${ }^{1}$ If $\mathbf{x}=\{\mathbf{r}, s\}$, where $s$ is the spin coordinate, the full spin-dependent density is

$$
\gamma(\mathbf{x})=\rho^{\alpha}(\mathbf{r})|\alpha(s)|^{2}+\rho^{\beta}(\mathbf{r})|\beta(s)|^{2}
$$

Integration (actually summation) over the spin coordinate $s$ gives the regular density

$$
\rho(\mathbf{r})=\int \gamma(\mathbf{x}) \mathrm{d} s=\rho^{\alpha}(\mathbf{r})+\rho^{\beta}(\mathbf{r})
$$

The normalization condition on $\gamma$ is

$$
\int \gamma(\mathbf{x}) \mathrm{d} \mathbf{x}=n
$$

where $n=n^{\alpha}+n^{\beta}$ is the total number of electrons.

Following the general principles of KS theory, ${ }^{8}$ the total electronic energy is

$$
E[\gamma]=T_{\mathrm{s}}[\gamma]+V_{\mathrm{ext}}[\gamma]+J[\gamma]+E_{\mathrm{XC}}[\gamma]
$$

Here, $V_{\text {ext }}[\gamma]$ is the potential energy in the field of the nuclei plus any external perturbation. In the absence of magnetic effects, this is $V_{\text {ext }}[\rho] . T_{\mathrm{s}}[\gamma]$ is the kinetic energy of a set of $n$ independent electrons, moving in an effective one-electron potential $v_{\text {eff }}(\mathbf{x})$, which leads to the prescribed density $\gamma(\mathbf{x}) . J[\gamma]$ is the total Coulomb interaction, given by 


$$
J[\gamma]=J[\rho]=\frac{1}{2} \iint \frac{\rho\left(\mathbf{r}_{1}\right) \rho\left(\mathbf{r}_{2}\right)}{r_{12}} \mathrm{~d} \mathbf{r}_{1} \mathrm{~d} \mathbf{r}_{2}
$$

Finally, $E_{\mathrm{XC}}[\gamma]$ is the remainder, usually described as the exchange-correlation energy. Equation 2.4 is essentially a definition of $E_{\mathrm{XC}}$.

Given a proposed approximate functional $E_{\mathrm{XC}}[\gamma]$, the KS treatment proceeds by writing $\gamma$ in terms of a set of orthonormal spin orbitals $\chi_{i}$

$$
\gamma(\mathbf{x})=\sum_{i=1}^{n}\left|\chi_{i}\right|^{2}
$$

The energy expression (2.4) is then minimized, subject to the orthonormality constraint, leading to the KS spin orbitals $\chi_{i}^{\mathrm{KS}}$ $(i=1, \ldots n)$. These satisfy the $\mathrm{KS}$ equations

$$
\left[-{ }^{1} / 2 \nabla^{2}+v_{\text {eff }}\right] \chi_{i}^{\mathrm{KS}}=\epsilon_{i} \chi_{i}^{\mathrm{KS}}
$$

where $\epsilon_{i}^{\mathrm{KS}}$ are the KS eigenvalues or one-electron energies.

In practice, the $\chi_{i}$ are usually expanded in a spin orbital basis, some integrations are carried out over specified grids, and further approximations may be made. However, we may develop the general theory before these are introduced.

At this point, we note that the HF procedure is a special case of KS density functional theory in which $E[\gamma]$ is the expectation value of the energy, using the single-determinant wave function built from the spin orbitals $\chi_{i}$, derived in turn from the density $\gamma$. This corresponds to the following "exchange-only" functional for $E_{\mathrm{XC}}[\gamma]$,

$$
E_{\mathrm{X}}[\gamma]=-\frac{1}{2} \sum_{i, j}^{n} \iint \frac{\chi_{i}^{*}(1) \chi_{j}(1) \chi_{j}^{*}(2) \chi_{i}(2)}{r_{12}}
$$

Note that this exchange energy may be defined by (2.8) for any set of appropriately normalized spin orbitals $\chi_{i}$ and hence for any appropriate $\gamma(\mathbf{x})$. We shall refer to $E_{\mathrm{X}}[\gamma]$ given by (2.8) as the "proper exchange energy" for $\gamma$ to distinguish it from other approximation forms. We call the latter "improper exchange energies", an example being the free-electron DiracSlater formula

$$
-\frac{3}{2}\left(\frac{3}{4 \pi}\right)^{1 / 3} \int\left[\left(\rho^{\alpha}\right)^{4 / 3}+\left(\rho^{\beta}\right)^{4 / 3}\right] \mathrm{d} \mathbf{r}
$$

In particular, we note that (2.7) and (2.8) together define a proper exchange energy for the KS orbitals. (The term "exact exchange energy" has also been used for this concept. We prefer to avoid this since there is a proper exchange energy even for approximate functionals.)

Returning to the general exchange-correlation functional $E_{\mathrm{XC}^{-}}$ $[\gamma]$, we may use the KS spin orbitals to form a singledeterminant wave function

$$
\Psi_{\mathrm{KS}}=\frac{1}{\sqrt{n}} \operatorname{det}\left[\chi_{1}^{\mathrm{KS}} \chi_{2}^{\mathrm{KS}} \cdots \chi_{n}^{\mathrm{KS}}\right]
$$

The expectation energy using this wave function will be

$$
E_{\mathrm{KS}}=T_{\mathrm{s}}\left[\lambda_{\mathrm{KS}}\right]+V_{\mathrm{ext}}\left[\gamma_{\mathrm{KS}}\right]+J\left[\gamma_{\mathrm{KS}}\right]+E_{\mathrm{X}}\left[\gamma_{\mathrm{KS}}\right]
$$

$E_{\mathrm{X}}$ being given by (2.8). The difference

$$
E_{\mathrm{C}}\left[\gamma_{\mathrm{KS}}\right]=E_{\mathrm{XC}}\left[\gamma_{\mathrm{KS}}\right]-E_{\mathrm{X}}\left[\gamma_{\mathrm{KS}}\right]
$$

may then be termed the "proper correlation energy" for the functional $E_{\mathrm{XC}}$.

We note that

$$
E_{\mathrm{KS}} \geq E_{\mathrm{HF}}
$$

since the HF energy is the lowest expectation energy that can be obtained from a single-determinant wave function. Also, from (2.12), $E_{\mathrm{C}}$ vanishes for the HF functional.

In the following discussion of partitioning using exchangecorrelation functionals, we will use the KS determinant (2.10) as the starting point for a conventional treatment of electron correlation. To this end, we need to introduce an orthonormal set of virtual spin orbitals $\chi_{a}^{\mathrm{KS}}$, all orthogonal to the occupied $\chi_{i}^{\mathrm{KS}}$. (We use the convention of suffices $i, j, \ldots$ for occupied, $a$, $b, \ldots$ for virtual, and $p, q, \ldots$ for generic spin orbitals.) If a finite basis is used, any such set of $\chi_{a}^{\mathrm{KS}}$ will suffice. We can then construct a Fock matrix for the KS determinant

$$
F_{p q}^{\mathrm{KS}}=T_{p q}+V_{p q}+\sum_{i=1}^{n}(p i \| q i)
$$

using the general notation for antisymmetrized two-electron integrals

$$
\begin{array}{r}
(p r \| q s)=\iint \chi_{p}(1) \chi_{r}(2) \frac{1}{r_{12}}\left[\chi_{q}(1) \chi_{s}(2)-\right. \\
\left.\chi_{s}(1) \chi_{q}(2)\right] \mathrm{d} \mathbf{x}_{1} \mathrm{~d} \mathbf{x}_{2}
\end{array}
$$

The Fock matrix (2.14) will not be diagonal, unless we are using the HF functional. In particular, there will be nonzero elements $F_{i a}$ connecting occupied and virtual spin orbitals. It is convenient to separate the full matrix (2.14) into two parts,

$$
F^{\mathrm{KS}}=F^{\mathrm{KS}}(\mathrm{OO}+\mathrm{VV})+F^{\mathrm{KS}}(\mathrm{OV})
$$

corresponding to nonzero occupied-occupied, virtual-virtual blocks in the first part and nonzero occupied-virtual blocks in the second. If the matrix $F^{\mathrm{KS}}(\mathrm{OO}+\mathrm{VV})$ is then diagonalized, we will obtain a new set of spinorbitals $\chi_{i}, \chi_{j}, \ldots, \chi_{a}, \chi_{b}, \ldots$ These could be described as "canonical Fock orbitals for the constrained Kohn-Sham determinant" (CFOCKSD). They will play a central role in the subsequent treatment and will be denoted by $\chi_{i}, \ldots, \chi_{a}, \ldots$ without further adornment. These new occupied spin orbitals will be an orthogonal transform of the KS occupied spin orbitals, i.e.

$$
\chi_{i}=\sum_{j=1}^{n} O_{i j} \chi_{j}^{K S}
$$

and will correspond to the same density

$$
\gamma=\sum_{i=1}^{n}\left|\chi_{i}\right|^{2}=\sum_{i=1}^{n}\left|\chi_{i}^{\mathrm{KS}}\right|^{2}=\gamma_{\mathrm{KS}}
$$

Each $\chi_{\mathrm{p}}$ will also be associated with an eigenvalue $\epsilon_{\mathrm{p}}$ of the blocked Fock matrix $F^{\mathrm{KS}}(\mathrm{OO}+\mathrm{VV})$. The single-determinant wave function formed from the occupied $\chi_{i}$ will be written $\Psi_{0}$; it is, of course, equal to $\Psi_{\mathrm{KS}}$.

We can now set up a conventional treatment of electron correlation as follows. A many-electron Hamiltonian is introduced with a connecting parameter $\lambda$

$$
\begin{aligned}
\mathbf{H}_{\lambda} & =\mathbf{H}_{0}+\lambda \mathbf{H}^{\prime} \\
& =\mathbf{H}_{0}+\lambda\left(\mathbf{H}-\mathbf{H}_{0}\right)
\end{aligned}
$$


where $\mathbf{H}$ is the full Hamiltonian and $\mathbf{H}_{0}$ is the zero-order sum of one-electron Fock operators $F(\mathrm{OO}+\mathrm{VV})$, for which $\Psi_{0}$ is an exact eigenfunction with eigenvalue $\sum \epsilon_{i}$. As $\lambda$ changes from 0 to 1 , the total energy will change from $\sum \epsilon_{i}$ to the exact answer (if there were no other approximations). To find the lowest eigenvalue of $\mathbf{H}$, starting from the determinant $\Psi_{0}$, we may proceed by various methods, such as configuration interaction, coupled-cluster theory, or Møller-Plesset perturbation theory. For the latter, the result is expanded in powers of $\lambda$ and the series truncated at some order. The final answer, if any of these techniques were carried out in full, would be

$$
E_{\text {exact }}=E_{\mathrm{KS}}+E_{\mathrm{C}}^{\mathrm{conv}}\left[\gamma_{\mathrm{KS}}\right]
$$

where the last term is the proper correlation energy for this particular KS starting point, evaluated by conventional means.

Alternatively, the exact energy could be split as

$$
E_{\text {exact }}=E_{\mathrm{NoXC}}\left[\gamma_{\mathrm{KS}}\right]+E_{\mathrm{X}}\left[\gamma_{\mathrm{KS}}\right]+E_{\mathrm{C}}^{\mathrm{conv}}\left[\gamma_{\mathrm{KS}}\right]
$$

where $E_{\mathrm{NoXC}}[\gamma]$ is the energy functional without exchange or correlation, as represented by the first three terms of (2.4), and $E_{\mathrm{X}}[\gamma]$ is given by the conventional (proper) formula (2.8). Since $E_{\mathrm{NoXC}}[\gamma]$ is identical in conventional and DFT theories, we could, in principle, test the full exchange-correlation functional by comparing $E_{\mathrm{X}}+E_{\mathrm{C}}^{\mathrm{conv}}$ in (2.21) with the DFT expression $E_{\mathrm{XC}}\left[\gamma_{\mathrm{KS}}\right]$. In practice, of course, both will have errors from additional approximations (finite basis sets, integration grids, etc.) which may hinder full agreement, but the objective should be kept in mind.

In this paper, the only conventional correlation method used is second-order Møller-Plesset theory (MP2). At first order, the energy is just the expectation value of $\mathbf{H}$ with $\Psi_{\mathrm{KS}}$

$$
E(\mathrm{MP} 1)=E_{\mathrm{KS}}=E_{\mathrm{NoXC}}\left[\gamma_{\mathrm{KS}}\right]+E_{\mathrm{X}}\left[\gamma_{\mathrm{KS}}\right]
$$

and the second-order correction

$$
E^{(2)}=-\sum_{i a} \frac{F_{i a}^{2}}{\epsilon_{a}-\epsilon_{i}}-\frac{1}{4} \sum_{i j a b} \frac{(i j \| a b)^{2}}{\epsilon_{a}+\epsilon_{b}-\epsilon_{i}-\epsilon_{j}}
$$

Note that $F_{i a}$ appears in (2.23) since the off-diagonal elements appear as part of the perturbation Hamiltonian $\mathbf{H}^{\prime}$ in (2.19). Equations 2.22 and 2.23 reduce to familiar forms if the $\mathrm{HF}$ functional is used, all $F_{i a}$ then vanishing. The first part of (2.23), involving the $F_{i a}$ elements, really allows for the mixing of occupied and virtual orbitals leading to a tendency for the KS orbitals to move toward HF, therefore modifying the density. If we suppose the density functional to give the correct density, it could be argued that these terms should be omitted, since any density changes introduced would have to be canceled in higher-order terms. The $F_{i a}$ contributions are much smaller than the second part of (2.23), and we shall omit them in the present treatment.

\section{Energy Partitions}

Suppose that the set of occupied spin orbitals $\chi_{i}$ is split into two nonoverlapping subsets ${ }^{\mathrm{A}} \chi_{i}$ and ${ }^{\mathrm{B}} \chi_{i}$. Since the density $\gamma$ is the sum of the squares of the spin orbitals (2.6), it will be consequently be partitioned into two parts ${ }^{\mathrm{A}} \gamma$ and ${ }^{\mathrm{B}} \gamma$ with

$$
\gamma(\mathbf{x})={ }^{\mathrm{A}} \gamma(\mathbf{x})+{ }^{\mathrm{B}} \gamma(\mathbf{x})
$$

Any energy functional $E\left[{ }^{\mathrm{A}} \gamma,{ }^{\mathrm{B}} \gamma\right]$ is then split into "pure A", "pure $\mathrm{B}$ ", and "interacting $\mathrm{AB}$ " parts by the partition

$$
\begin{gathered}
{ }^{\mathrm{A}} E=E\left[{ }^{\mathrm{A}} \gamma, 0\right] \\
{ }^{\mathrm{B}} E=E\left[0,{ }^{\mathrm{B}} \gamma\right] \\
{ }^{\mathrm{AB}} E=E\left[{ }^{\mathrm{A}} \gamma,{ }^{\mathrm{B}} \gamma\right]-{ }^{\mathrm{A}} E-{ }^{\mathrm{B}} E
\end{gathered}
$$

This kind of partition was proposed and used for spin components of the correlation energy by Stoll et al. ${ }^{4}$ and Perdew et al., ${ }^{5}$ but can be applied elsewhere. On the conventional side, we may treat the energies as functionals of the sets ${ }^{\mathrm{A}} \chi$ and ${ }^{\mathrm{B}} \chi$ and use a similar partition

$$
\begin{gathered}
{ }^{\mathrm{A}} E=E\left[{ }^{\mathrm{A}} \chi_{i}, 0\right] \\
{ }^{\mathrm{B}} E=E\left[0,{ }^{\mathrm{B}} \chi_{i}\right] \\
{ }^{\mathrm{AB}} E=E\left[{ }^{\mathrm{A}} \chi_{i},{ }^{\mathrm{B}} \chi_{i}\right]-{ }^{\mathrm{A}} E-{ }^{\mathrm{B}} E
\end{gathered}
$$

Here $E\left[{ }^{\mathrm{A}} \chi_{i}, 0\right]$ means that all integrals involving B spin orbitals are zeroed in matrix elements of the Hamiltonian $\mathbf{H}^{\prime}$ in (2.19).

When applied to the second-order Møller-Plesset correlation energy (2.23), this partition cleanly separates the full energy into separate pair terms. The same applies to the computation of the proper exchange energy (2.8) where the total is also strictly separable into pair terms.

It may be questioned whether the partitions (3.2) and (3.3) are strictly comparable for computation of conventional correlation energies at higher levels (up to full configuration interaction). There are many complicated many-body interactions involved, which are somewhat arbitrarily assigned by (3.3). However, we shall not address this question here, since only MP2 computations are performed. It seems reasonable that the DFT and conventional partitions (3.2) and (3.3) should be comparable at a coarse level, since MP2 usually accounts for most of the correlation in simple pair terms.

As noted in the Introduction, we will partition the density and spin orbitals in two ways. The subsets A and B may be the $\alpha$ and $\beta$ occupied spin orbitals, or they may be the core and valence $(K, L)$ spin orbitals if we are separating correlation of valence electrons from the rest. The procedures used are closely related.

\section{Applications}

The partition schemes described in the previous section have been implemented in the Q-Chem program $^{2 a}$ by one of us (R.D.A.). As a preliminary application, we have tested the LSD (free-electron gas) functional for the first-row atoms and the molecules $\mathrm{H}_{2}, \mathrm{~N}_{2}, \mathrm{~F}_{2}, \mathrm{FH}, \mathrm{OH}_{2}, \mathrm{NH}_{3}$, and $\mathrm{CH}_{4}$.

The orbital basis used is $6-311+\mathrm{G}(3 \mathrm{df}, 2 \mathrm{p})$, the largest basis present in G2 theory. ${ }^{9}$ The actual LSD functional is DiracSlater for exchange ${ }^{10}$ and Vosko-Wilk-Nusair for correlation. ${ }^{11}$ Quadrature is performed using the standard SG-1 grid. ${ }^{12}$ Geometries are as in G2 theory. The individual energies may be identified as

$$
\text { functional/LSD/6-311+G(3df,2p)//MP2(full)6-31G(d) }
$$

meaning that the functional is applied to the LSD density, using the $6-311+\mathrm{G}(3 \mathrm{df}, 2 \mathrm{p})$ density, at the MP2/6-31G(d) geometries.

The total energies are listed in Table 1. The first column gives the energies obtained by solving the Kohn-Sham equations for the LSD functional. The other columns are energies using this density. In particular, the final column gives the expectation energy, using the KS single determinant. This is $E_{\mathrm{KS}}$ as given by (2.11). 
TABLE 1: Total Energies ${ }^{a}$ (hartrees)

\begin{tabular}{lrrrr}
\hline & \multicolumn{1}{c}{ LSD } & \multicolumn{1}{c}{ NoXC } & \multicolumn{1}{c}{ HFS } & \multicolumn{1}{c}{$E_{\mathrm{KS}}$} \\
\hline $\mathrm{H}$ & -0.47835 & -0.19921 & -0.45663 & -0.49894 \\
$\mathrm{He}$ & -2.83256 & -1.85619 & -2.72081 & -2.85821 \\
$\mathrm{Li}$ & -7.34221 & -5.67152 & -7.19113 & -7.43001 \\
$\mathrm{Be}$ & -14.44484 & -11.92421 & -14.22024 & -14.56933 \\
$\mathrm{~B}$ & -24.35242 & -20.79262 & -24.06338 & -24.52534 \\
$\mathrm{C}$ & -37.46510 & -34.65274 & -37.10778 & -37.68297 \\
$\mathrm{~N}$ & -54.12937 & -47.83831 & -53.70141 & -54.39175 \\
$\mathrm{O}$ & -74.51998 & -66.65201 & -73.98786 & -74.79881 \\
$\mathrm{~F}$ & -99.09882 & -89.42861 & -98.46121 & -99.38934 \\
$\mathrm{Ne}$ & -128.21133 & -116.49864 & -127.46811 & -128.51382 \\
$\mathrm{H}$ & -1.13674 & -0.48254 & -1.04256 & -1.13229 \\
$\mathrm{~N}$ & -108.68189 & -95.94700 & -107.73911 & -108.94796 \\
$\mathrm{~F}_{2}$ & -198.31988 & -178.89857 & -197.02225 & -198.70752 \\
$\mathrm{FH}$ & -99.83580 & -89.70121 & -99.13477 & -100.04198 \\
$\mathrm{OH}_{2}$ & -75.90141 & -67.16846 & -75.24027 & -76.04380 \\
$\mathrm{NH}_{3}$ & -56.10183 & -48.59154 & -55.47729 & -56.20576 \\
$\mathrm{CH}_{4}$ & -40.11538 & -33.66071 & -39.52360 & -40.19840
\end{tabular}

${ }^{a}$ All energies evaluated using the LSD density.

TABLE 2: $\quad$ Energy Differences $\boldsymbol{E}_{\mathrm{KS}}-\boldsymbol{E}_{\mathrm{HF}}$ (mhartrees)

$\begin{array}{lllr}\mathrm{H} & 0.87 & \mathrm{Ne} & 12.66 \\ \mathrm{He} & 1.69 & \mathrm{H}_{2} & 0.71 \\ \mathrm{Li} & 2.02 & \mathrm{~N}_{2} & 24.59 \\ \mathrm{Be} & 2.61 & \mathrm{~F}_{2} & 33.78 \\ \mathrm{~B} & 5.74 & \mathrm{FH} & 13.35 \\ \mathrm{C} & 7.28 & \mathrm{OH}_{2} & 12.72 \\ \mathrm{~N} & 7.14 & \mathrm{NH}_{3} & 12.40 \\ \mathrm{O} & 10.53 & \mathrm{CH}_{4} & 13.82 \\ \mathrm{~F} & 12.47 & & \end{array}$

TABLE 3: Spin Components of Correlation Energy (mhartrees)

\begin{tabular}{|c|c|c|c|c|c|c|}
\hline & \multicolumn{3}{|c|}{ DFT (LSD) } & \multicolumn{3}{|c|}{ conventional (MP2) } \\
\hline & $\alpha \alpha$ & $\beta \beta$ & $\alpha \beta$ & $\alpha \alpha$ & $\beta \beta$ & $\alpha \beta$ \\
\hline $\mathrm{H}$ & 21.72 & 0 & 0 & 0 & 0 & 0 \\
\hline $\mathrm{He}$ & 26.89 & 26.89 & 57.96 & 0 & 0 & 29.61 \\
\hline $\mathrm{Li}$ & 47.12 & 32.36 & 71.59 & 0.16 & 0 & 13.43 \\
\hline $\mathrm{Be}$ & 54.20 & 54.20 & 116.21 & 0.25 & 0.25 & 43.13 \\
\hline B & 83.70 & 59.77 & 145 & 4.29 & 0.33 & 58.84 \\
\hline $\mathrm{C}$ & 118.35 & 64.26 & 174 & 14.81 & 0.36 & 72.95 \\
\hline $\mathrm{N}$ & 3 & 68. & 202 & 32.76 & 0.3 & 85.06 \\
\hline $\mathrm{O}$ & 165.71 & 101. & 265 & 34.75 & 5.4 & 129.71 \\
\hline $\mathrm{F}$ & 173.04 & 138.46 & 326.11 & 36.68 & 18.05 & 175.56 \\
\hline $\mathrm{Ne}$ & 179.45 & 179.45 & 384.32 & 38.51 & 38.51 & 221.25 \\
\hline $\mathrm{H}_{2}$ & 22.66 & 22.66 & 48.85 & 0 & 0 & 29.87 \\
\hline $\mathrm{N}_{2}$ & 227.41 & 227.41 & 487.97 & 56.32 & 56.32 & 339.76 \\
\hline $\mathrm{F}_{2}$ & 313.18 & 313.18 & 671.27 & 75.10 & 75.10 & 456.55 \\
\hline $\mathrm{FH}$ & 169.16 & 169.16 & 362.71 & 38.91 & 38.91 & 230.33 \\
\hline $\mathrm{OH}_{2}$ & 159.44 & 159.44 & 342.25 & 35.58 & 35.58 & 223.61 \\
\hline $\mathrm{NH}_{3}$ & 150.54 & 150.54 & 323.46 & 29.30 & 29.30 & 204.97 \\
\hline $\mathrm{CH}_{4}$ & 142.58 & 142.58 & 306.62 & 21.15 & 21.15 & 180.98 \\
\hline
\end{tabular}

We note that $E_{\mathrm{KS}} \geq E_{\mathrm{HF}}$; see (2.13). The differences $E_{\mathrm{KS}}-$ $E_{\mathrm{HF}}$, which are listed in Table 2, follow from the final column of Table 1 and regular HF calculations with the same orbital basis and geometry. They arise because of differences between the LSD and HF densities. The magnitudes of these differences are small compared with correlation energies, but clearly not negligible. They must be taken into account in comparing DFT correlation energies with conventional values based on a HF starting point.

We next turn to the partitions of the correlation energy discussed in this paper. Table 3 shows the spin partitions $(\alpha \alpha, \beta \beta, \alpha \beta)$ for the LSD functional using (3.2) with $A=\alpha$ and $B=\beta$. It also shows the conventional MP2 correlation energies with the KS starting point, using (2.23) (without the first term) and (3.3). It should be noted that these are full correlation energies, taking account of all electron pairs. Table 4 gives the DFT correlation energies, split into core-core, core- valence, and valence-valence parts (eq 3.2 with $A=K, B=$ $L$ ); the total is also listed in the first column. The table also shows valence-valence correlation energies by conventional MP2 theory, again with the KS starting point. We do not show the conventional core-core and core-valence pieces; their significance would be limited as the orbital basis $6-311+\mathrm{G}-$ (3df,2p) permits only a very poor description of inner-shell correlation. Finally, in the last column of Table 4, we give a list of "best" valence-valence results for comparative purposes. These are obtained from QCISD(T) results using regular correlation theory based on HF starting point (these are used in the general $\mathrm{G} 2(\mathrm{QCI}) \operatorname{model}^{9}$ ) but are incremented by the $\Delta$ values from Table 2, thereby allowing for the KS reference.

In view of the inadequate nature of the orbital basis in the inner-shell regions of the atoms and molecules, we also present a further partition of the valence correlation into $\alpha \alpha, \beta \beta$ and $\alpha \beta$ spin components. This is done by straightforward application of the partition (3.2) $(A=\alpha, B=\beta)$ to the functional $E_{\mathrm{C}^{-}}$ $\left[\gamma_{\text {valence }}\right]$. These results are listed in Table 5, together with the corresponding MP2 partitions.

\section{Discussion}

The total correlation energies, listed in Table 4, demonstrate the familiar overestimation by the LSD functional. These results are close to previously published values. ${ }^{13}$ Correct total atomic correlation energies, relative to a HF reference, are known to range from 42 mhartrees for helium to 392 mhartrees for neon. ${ }^{14}$ The overestimate is roughly by a factor of 2 .

Now consider the spin components of correlation energy listed in Table 3. These results are closely related to early work by Stoll et al. ${ }^{4}$ They partitioned the LSD correlation energy in this manner, but then compared just the $\alpha \beta$ component with total correlation energies from conventional theory. However, the $\alpha \alpha$ and $\beta \beta$ components of $E_{\mathrm{C}}(\mathrm{LSD})$ are clearly large, as shown in the first two columns. Such spin-parallel contributions are partly spurious, as indicated by significant nonzero values for $\mathrm{H}, \mathrm{He}$, and $\mathrm{H}_{2}$. These represent a nonexistent self-correlation of single electrons. Such effects undoubtedly also contribute to the very large $\alpha \alpha$ and $\beta \beta$ values for the other systems, which are far greater than the corresponding conventional results also shown in Table 3, even allowing for the core effects which are poorly treated in the conventional calculations. Spin-parallel correlation effects (beyond those implicit in the exchange term) are clearly significant, but not as large as given by the LSD functional.

The spin-antiparallel $\alpha \beta$ components, shown in Table 3, are still significantly larger for the LSD functional than for the conventional (MP2) method. For the helium atom, there is only $\alpha \beta$ correlation, and the LSD value (58.0 mhartrees) exceeds the known accurate value (43.7 mhartrees after applying the 1.7 mhartrees correction given in Table 2), but no longer by a factor of 2. For the larger systems, there is greater overestimation, but we note that the MP2 results will be underestimates, partly because the orbital basis used is very crude in the innershell region and will not well describe this part of the atom or molecule. We will return to this matter below.

It is interesting to compare the contributions of parallel and antiparallel correlation to chemical binding energies. These are shown in Table 6, for DFT and conventional methods, together with corresponding total correlation bindings. It is well recognized that electron correlation plays a major role in determining bond energies. The total MP2 correlation binding energies listed in the final column reflect this. The DFT totals are mostly lower $\left(\mathrm{H}_{2}\right.$ and $\mathrm{CH}_{4}$ being exceptions), $\mathrm{F}_{2}$ being remarkably lower. When broken down into spin components, 
TABLE 4: Core-Valence Components of Correlation Energy (mhartrees)

\begin{tabular}{|c|c|c|c|c|c|c|c|}
\hline & \multicolumn{4}{|c|}{ DFT (LSD) } & \multicolumn{3}{|c|}{ conventional } \\
\hline & total & core & core/valence & valence & valence (MP2) & valence (best) & core $\left(\right.$ best $\left.^{a}\right)$ \\
\hline $\mathrm{H}$ & 21.72 & 0 & 0 & 21.72 & 0 & 0 & 0 \\
\hline $\mathrm{He}$ & 111.75 & 0 & 0 & 111.75 & 29.61 & 37.57 & 0 \\
\hline $\mathrm{Li}$ & 151.08 & 134.28 & 2.61 & 14.18 & 0 & 0 & 43.50 \\
\hline $\mathrm{Be}$ & 224.60 & 150.22 & 3.01 & 71.38 & 28.10 & 48.04 & 44.27 \\
\hline B & 289.04 & 162.73 & 5.27 & 121.04 & 45.67 & 71.74 & 44.74 \\
\hline $\mathrm{C}$ & 357.32 & 173.02 & 8.23 & 176.08 & 68.63 & 96.22 & 45.05 \\
\hline $\mathrm{N}$ & 427.96 & 181.76 & 11.59 & 234.60 & 97.22 & 120.97 & 45.28 \\
\hline $\mathrm{O}$ & 532.11 & 189.37 & 10.72 & 332.03 & 147.87 & 173.22 & 45.45 \\
\hline $\mathrm{F}$ & 637.60 & 196.10 & 10.51 & 430.99 & 207.12 & 228.48 & 45.59 \\
\hline $\mathrm{Ne}$ & 743.22 & 202.14 & 10.93 & 530.15 & 273.92 & 285.20 & 45.69 \\
\hline $\mathrm{H}_{2}$ & 94.18 & 0 & 0 & 94.18 & 29.87 & 38.52 & 0 \\
\hline $\mathrm{N}_{2}$ & 942.79 & 363.35 & 15.49 & 563.95 & 407.24 & 424.44 & \\
\hline $\mathrm{F}_{2}$ & 1297.62 & 392.17 & 19.70 & 885.75 & 559.73 & 582.74 & \\
\hline $\mathrm{FH}$ & 701.03 & 196.08 & 9.92 & 495.03 & 284.32 & 296.02 & \\
\hline $\mathrm{OH}_{2}$ & 661.14 & 189.34 & 8.87 & 462.93 & 271.61 & 288.76 & \\
\hline $\mathrm{NH}_{3}$ & 624.54 & 181.71 & 7.80 & 435.03 & 240.67 & 265.37 & \\
\hline $\mathrm{CH}_{4}$ & 591.77 & 172.90 & 6.74 & 412.13 & 200.65 & 234.61 & \\
\hline
\end{tabular}

${ }^{a}$ From ref 14.

TABLE 5: Spin Components of Valence-Valence Correlation Energy (mhartrees)

\begin{tabular}{lrrrrrrr}
\hline & \multicolumn{3}{c}{ DFT (LSD) } & & \multicolumn{3}{c}{ conventional (MP2) } \\
\cline { 2 - 3 } \cline { 7 - 8 } & $\alpha \alpha$ & $\beta \beta$ & $\alpha \beta$ & & $\alpha \alpha$ & $\beta \beta$ & $\alpha \beta$ \\
\hline $\mathrm{Li}$ & 14.18 & 0 & 0 & & 0 & 0 & 0 \\
$\mathrm{Be}$ & 17.23 & 17.23 & 36.93 & & 0 & 0 & 28.10 \\
$\mathrm{~B}$ & 43.08 & 19.63 & 58.33 & & 3.50 & 0 & 42.16 \\
$\mathrm{C}$ & 74.61 & 21.53 & 79.93 & & 13.41 & 0 & 55.22 \\
$\mathrm{~N}$ & 110.59 & 23.14 & 100.87 & & 30.74 & 0 & 66.48 \\
$\mathrm{O}$ & 117.26 & 53.60 & 161.17 & & 32.68 & 4.49 & 110.71 \\
$\mathrm{~F}$ & 122.84 & 88.75 & 219.40 & & 34.55 & 16.48 & 156.10 \\
$\mathrm{Ne}$ & 127.70 & 127.70 & 274.75 & & 36.33 & 36.33 & 201.25 \\
$N_{2}$ & 135.65 & 135.65 & 292.64 & & 53.19 & 53.19 & 300.86 \\
$F_{2}$ & 213.23 & 213.23 & 459.29 & & 71.25 & 71.25 & 417.24 \\
$\mathrm{FH}$ & 119.17 & 119.17 & 256.69 & & 36.89 & 36.89 & 210.54 \\
$\mathrm{OH}_{2}$ & 111.39 & 111.39 & 240.16 & & 33.76 & 33.76 & 204.09 \\
$\mathrm{NH}_{3}$ & 104.64 & 104.64 & 225.75 & & 27.62 & 27.62 & 185.44 \\
$\mathrm{CH}_{4}$ & 99.12 & 99.12 & 213.90 & & 19.60 & 19.60 & 161.45
\end{tabular}

TABLE 6: Spin Components of Correlation Binding Energy (mhartrees)

\begin{tabular}{lcccccrrr}
\hline & \multicolumn{3}{c}{ DFT (LSD) } & & \multicolumn{3}{c}{ conventional (MP2) } \\
\cline { 2 - 3 } \cline { 7 - 8 } & $\alpha \alpha+\beta \beta$ & $\alpha \beta$ & total & & $\alpha \alpha+\beta \beta$ & $\alpha \beta$ & \multicolumn{1}{c}{ total } \\
\hline $\mathrm{H}_{2}$ & 1.88 & 48.85 & 50.74 & & 0 & 29.87 & 29.87 \\
$\mathrm{~N}_{2}$ & 4.64 & 82.23 & 86.87 & & 46.37 & 169.64 & 216.01 \\
$\mathrm{~F}_{2}$ & 3.37 & 19.05 & 22.41 & & 40.74 & 105.44 & 146.18 \\
$\mathrm{FH}$ & 5.10 & 36.60 & 41.70 & & 23.09 & 54.77 & 77.86 \\
$\mathrm{OH}_{2}$ & 8.69 & 76.89 & 85.58 & & 30.96 & 93.89 & 124.85 \\
$\mathrm{NH}_{3}$ & 10.83 & 120.59 & 131.42 & & 25.46 & 119.91 & 145.37 \\
$\mathrm{CH}_{4}$ & 15.65 & 131.91 & 147.56 & & 27.13 & 108.03 & 135.16
\end{tabular}

the contributions of parallel spins are seen to be quite small by LSD (in spite of the large individual $\alpha \alpha$ and $\beta \beta$ values listed in Table 3). The MP2 calculations indicate larger parallel contributions (except $\mathrm{H}_{2}$, where there is none), although most of the correlation binding clearly comes from the antiparallel parts. The $\alpha \beta$ correlation bindings themselves (columns 2 and 3 in Table 3) show some improvement when compared with the general overestimation of total correlation energies by a factor of 2. However, there are wide variations with the type of bond. For bonds to hydrogen, $\alpha \beta$ correlation binding is described moderately well by LSD. The triple bond in $\mathrm{N}_{2}$, on the other hand, for which correlation between three $\alpha \beta$ pairs is known to be a major stabilizing factor, shows $\alpha \beta$ underestimation by LSD by a factor of more than 2. This is particularly disturbing, since total correlation energies (Table 4) are overestimated by factors of this order. For the $F_{2}$ molecule, the $\alpha \beta$ correlation is also badly underestimated by the LSD functional. The bond in $\mathrm{F}_{2}$ is quite weak and exists only by virtue of electron correlation, since unrestricted HF theory gives a repulsive potential curve. The full LSD energy does give binding for $F_{2}$ (122 mhartrees from the energies in Table 1), but this arises mainly from the exchange part (HFS giving binding of 99 mhartrees).

Now we turn to core/valence separation results, listed in Table 4. We note first that the LSD functional gives a fairly good separation into core-core and valence-valence parts but that the LSD core-core correlation energies are much too large. Although we are not able to get an adequate conventional MP2 description of atomic core-core correlation with this orbital basis, we expect that the values would be close to those for the corresponding two-electron ions $\left(\mathrm{He}, \mathrm{Li}^{+}, \mathrm{Be}^{2+}, \mathrm{B}^{3+}, \ldots\right)$. On the other hand, it is known that these remain fairly constant and approach a limit of about 46 mhartrees $^{14}$ as shown in the final column. The LSD values are larger by a factor of up to 4 and increase steadily. Clearly, this is a major contribution to the overestimation of total correlation energies by the LSD functional.

The cross core-valence $(K L)$ parts are relatively small, consistent with the expectation that the corresponding spin orbitals are principally located in different spatial regions. However, we cannot compare with good conventional numbers with our limited basis set. There has been little other work on these components.

The valence-valence LSD correlations listed in Table 4 are also too large, by a factor of about 2 . This is partly due to the inadequate conventional correlation level (MP2), but comparison with the better results in the final column gives only relatively small improvements.

One positive feature of the LSD results in Table 4 is that core-core correlation components do carry their large errors as a constant from atom to molecule. Thus, the failure to give an adequate description of the inner-shell electrons is not related to any failures in describing chemical bonding. The same is true for core-valence components, which also remain approximately constant from atom to molecule.

The good LSD core-valence separation found in Table 4, and the insensitivity of chemically significant results to core errors, suggest that we should examine valence-only LSD theory in more detail to evaluate its behavior. We therefore turn to the spin-component analysis of the valence correlation energies, summarized in Table 5. Here we are able to make a more 
TABLE 7: Spin Components of Valence Correlation Binding Energy (mhartrees)

\begin{tabular}{lcccccrr}
\hline & \multicolumn{3}{c}{ DFT (LSD) } & & \multicolumn{3}{c}{ conventional (MP2) } \\
\cline { 2 - 3 } \cline { 7 - 8 } & $\alpha \alpha+\beta \beta$ & $\alpha \beta$ & total & & $\alpha \alpha+\beta \beta$ & $\alpha \beta$ & total \\
\hline $\mathrm{N}_{2}$ & 3.85 & 90.89 & 94.74 & & 44.89 & 167.90 & 212.79 \\
$\mathrm{~F}_{2}$ & 3.28 & 20.49 & 23.77 & & 40.45 & 105.04 & 145.48 \\
$\mathrm{FH}$ & 5.02 & 37.29 & 42.31 & & 22.76 & 54.44 & 77.20 \\
$\mathrm{OH}_{2}$ & 8.47 & 78.99 & 87.46 & & 30.36 & 93.38 & 123.73 \\
$\mathrm{NH}_{3}$ & 10.39 & 124.88 & 135.27 & & 24.49 & 118.95 & 143.44 \\
$\mathrm{CH}_{4}$ & 15.20 & 133.97 & 149.17 & & 25.80 & 106.23 & 132.02
\end{tabular}

TABLE 8: Spin Components of Correlation Ionization Potentials (mhartrees)

\begin{tabular}{lccccccc}
\hline & \multicolumn{3}{c}{ DFT (LSD) } & & \multicolumn{3}{c}{ conventional (MP2) } \\
\cline { 2 - 3 } \cline { 7 - 8 } & $\alpha \alpha+\beta \beta$ & $\alpha \beta$ & total & & $\alpha \alpha+\beta \beta$ & $\alpha \beta$ & total \\
\hline $\mathrm{N}_{2}$ & 24.92 & 32.25 & 57.17 & & -12.32 & -8.86 & -21.18 \\
$\mathrm{FH}$ & 26.00 & 36.08 & 62.08 & & 24.02 & 21.22 & 45.24 \\
$\mathrm{OH}_{2}$ & 24.27 & 34.31 & 58.58 & & 21.43 & 19.78 & 41.21 \\
$\mathrm{NH}_{3}$ & 22.43 & 32.58 & 55.01 & & 17.27 & 17.14 & 34.41 \\
$\mathrm{CH}_{4}$ & 23.33 & 32.20 & 55.53 & & 9.45 & 11.49 & 20.93
\end{tabular}

satisfactory comparison with conventional MP2 results since the orbital basis is of high quality in the valence region.

The spin-parallel $(\alpha \alpha$ and $\beta \beta)$ components of valence correlation energies are still too large and still show the spurious self-interaction effects (as in $\mathrm{Li}$ and $\mathrm{Be}$, where such terms should vanish). On the other hand, the comparison of the LSD $\alpha \beta$ valence terms with conventional values (columns 3 and 6 in Table 5) shows a significant improvement over the all-electron results. The LSD results are still mostly too large, but now by a factor of only about 1.5 , rather than 2 as before.

We can also analyze the spin components of the correlation energy to the binding energy, using only the valence electron density. These can be found from Table 5, yielding the results in Table 7. Clearly, the performance of valence-only theory in this regard is very similar to the all-electron results of Table 6. The correlation bindings are mostly too small and are incorrectly divided between parallel and antiparallel components. This is consistent, of course, with a good cancellation of inner-shell contributions between atoms and molecules as noted above.

Finally, in Table 8, we present spin components of the correlation energy to ionization energies. The total LSD correlation contributions to the ionization of the five molecules studied are consistently around 60 mhartrees and bear little resemblance to the conventional values. The LSD spin analysis implies that most of the total contribution comes from the antiparallel component whereas the conventional analysis indicates roughly equal contributions from $\alpha \alpha+\beta \beta$ and $\alpha \beta$.

\section{Conclusions}

The following conclusions may reasonably be drawn from this work: (1) Density functional correlation energies can be compared in a partitioned manner with corresponding energies obtained by conventional theories. This may be done by using the Kohn-Sham single determinant as a reference. (2) The spin orbital or density partitions, core/valence and $\alpha \operatorname{spin} / \beta$ spin, may be implemented using the same general procedure. In this work, we have only considered partition of conventional correlation energies at the MP2 perturbation level. (3) When applied to the local density (LSD) energy function, the spin partition shows strong overestimation of $\alpha \alpha+\beta \beta$ correlation (including well-known spurious one-electron effects). The $\alpha \beta$ correlation is also overestimated but by a smaller factor. (4) The core/valence partition of the LSD correlation energy shows core values which are much too large. The numbers also fail to approach a limiting value, as they should. On the other hand, these core errors are fairly constant between atoms and molecules, leading to good systematic cancellation (as already noted for conventional theories). (5) When the LSD functional is applied to valence electrons only, the spin partition continues to show major errors for the $\alpha \alpha+\beta \beta$ parts; the $\alpha \beta$ components, however, are now overestimated by a factor of only about 1.5. (6) The relative contributions of $\alpha \alpha+\beta \beta$ and $\alpha \beta$ correlation to chemical bond energies are poorly given by the LSD functional. In particular, the contribution of antiparallel spins to correlation binding is usually too low, even though the overall binding energies calculated at the LAD level are generally too high.

The theory developed above is applicable to any energy functional and should permit a more detailed evaluation of the many that are available. We will present such an evaluation elsewhere. ${ }^{6}$ It is to be hoped that such studies of the pieceby-piece inadequacies will help in the search for systematic convergence toward the ultimate, correct energy functional.

Acknowledgment. This work was presented at the "Molecular Quantum Mechanics" conference held in memory of S.F. Boys and honor of I. Shavitt at Cambridge University (Sept $3-7,1995)$ and was partly supported by the National Science Foundation (Grant 8918623) and the NZ Lottery Grants Board (Grant AP-32002).

\section{References and Notes}

(1) Parr, R. G.; Yang, W. Density-Functional Theory of Atoms and Molecules; Oxford: New York, 1989.

(2) (a) Johnson, B. G.; Gill, P. M. W.; Head-Gordon, M.; White, C. A.; Maurice, D. R.; Adamson, R. D.; Adams, T. R.; Oumi, M. Q-Chem; Q-Chem Inc.: Pittsburgh, PA, 1995. (b) Amos, R. D.; Alberts, I. L.; Andrews, J. S.; Colwell, S. M.; Handy, N. C.; Jayatilaka, D.; Knowles, P. J.; Kobayashi, R.; Koga, N.; Laidig, K. E.; Maslen, P. E.; Murray, C. W.; Rice, J. E.; Sanz, J.; Simandiras, E. D.; Stone, A. J.; Su, M.-D. Cadpac 5, Cambridge, 1992.

(3) Gill, P. M. W.; Johnson, B. G.; Pople, J. A.; Frisch, M. J. Int. J. Quantum Chem. 1992, 26, 319.

(4) (a) Stoll, H.; Pavlidou, C. M. E.; Preuss, H. Theor. Chim. Acta 1978, 49, 143. (b) Stoll, H.; Golka, E.; Preuss, H. Theor. Chim. Acta 1980, $55,29$.

(5) Perdew, J. P.; McMullen, E. R.; Zunger, A. Phys. Rev. A 1981, 23, 2785.

(6) Pople, J. A.; Adamson, R. D.; Gill, P. M. W. J. Chem. Phys., in press.

(7) Löwdin, P.-O. Phys. Rev. 1955, 97, 1474.

(8) Kohn, W.; Sham, L. J. Phys. Rev. A 1965, 140, 1133.

(9) Curtiss, L. A.; Carpenter, J. E.; Raghavachari, K.; Pople, J. A. J. Chem. Phys. 1992, 96, 9030.

(10) Dirac, P. A. M. Proc. Cambridge Philos. Soc. 1930, 26, 376.

(11) Vosko, S. H.; Wilk, L.; Nusair, M. Can. J. Phys. 1980, 58, 1200.

(12) Gill, P. M. W.; Johnson, B. G.; Pople, J. A. Chem. Phys. Lett. 1993, 209, 506.

(13) Becke, A. D. J. Chem. Phys. 1992, 96, 2155.

(14) Davidson, E. R.; Hagstrom, S. A.; Chakravorty, S. J.; Umar, V. M.; Fischer, C. Phys. Rev. A 1991, 44, 7071.

JP963467Y 